\title{
PERCEPÇÃO DA MULHER ACERCA DA ASSISTÊNCIA AO PARTO PELA ENFERMEIRA OBSTETRA
}

Amanda Bezerra ${ }^{1}$, Nayale Lucinda Andrade de Albuquerque 2 , Ana Clara Silva Carvalho ${ }^{3}$, Renato Daniel Melo da Silva ${ }^{4}$ Cynthia Dantas Vicente ${ }^{4}$

Objetivo: Identificar a percepção das mulheres acerca da assistência ao parto pela enfermeira obstetra. Metodologia: estudo descritivo, exploratório, qualitativo, realizado em Recife/PE, Brasil, no ano de 2016 com 28 puérperas que tiveram seus partos assistidos por enfermeiras obstetras. Foram realizadas entrevistas semi-estruturadas, guiada pela questão norteadora: Qual a sua visão sobre a assistência da enfermeira obstetra que acompanhou seu parto? As falas foram submetidas à análise de conteúdo temática proposta por Bardin. Resultados: a partir da análise das falas, surgiram duas categorias temáticas: Assistência da enfermeira obstetra e experiência de partos anteriores. Considerações finais: Embora a enfermagem obstétrica tenha expandido e a sua assistência satisfatória, a baixa qualidade das informações transmitidas nas consultas de pré-natal e o pouco conhecimento da atuação deste ramo da enfermagem, demonstra a necessidade de se difundir cada vez mais esta profissão nos diferentes setores da sociedade e niveis de complexidades da área de educação e saúde.

DESCRITORES: Cuidados de enfermagem; Enfermagem; Gestantes; Obstetrícia; Parto.

\section{WOMEN'S PERCEPTION OF OBSTETRIC NURSE ASSISTANCE}

Objective: To identify the perception of women about delivery care by the obstetrician nurse. Methodology: a descriptive, exploratory, qualitative study was carried out in Recife / PE, Brazil, in the year 2016, with 28 postpartum women attended by obstetrical nurses. Semi-structured interviews were conducted, guided by the guiding question: What is your view about the assistance of the obstetrician nurse that accompanied your delivery? The speeches were submitted to the thematic content analysis proposed by Bardin. Results: based on the analysis of the speech, two thematic categories emerged: Obstetric nurse care and previous delivery experience. Conclusion: Although obstetrical nursing has expanded and its attendance satisfactory, the low quality of the information transmitted in the prenatal consultations and the little knowledge of the nursing activity, demonstrates the need to spread this profession in different ways. Sectors of society and complexity levels in the area of education and health.

DESCRIPTORS: Nursing Care; Nursing Pregnant Women; Obstetrics; Parturition

\section{PERCEPCIÓN DE LA MUJER ACERCA DE LAASISTENCIAAL PARTO POR LA ENFERMERA OBSTETRA}

Objetivo: identificar la percepción de las mujeres sobre la atención del parto por partera. Metodología: Se realizó un estudio descriptivo, exploratorio cualitativo realizado en Recife / PE, Brasil, en el año 2016 con 28 madres que tuvieron sus partos atendidos por parteras. Las entrevistas fueron semi-estructurado, guiado por la pregunta: ¿Cuál es su opinión sobre la asistencia de la enfermera obstétrica que acompañó a su nacimiento? Los informes fueron sometidos a análisis de contenido temático propuesto por Bardin. Resultados: A partir del análisis de los discursos, dos categorías temáticas surgieron: la asistencia enfermera obstetra y la experiencia de anteriores entregas. Conclusiónes: A pesar de la obstetricia se ha expandido y su servicio satisfactorio, la baja calidad de la información transmitida en las consultas prenatales y poco conocimiento de la realización de esta rama de enfermeria, demuestra la necesidad de difundir más y más la profesión en los distintos sectores de la sociedad y los niveles de educación y salud complejidades.

PALABRAS CLAVE: Los cuidados de enfermeira; Enfermería; Las mujeres embarazadas; Obstetricia; Entrega. 


\section{INTRODUÇÃO}

Os rituais que cercam o parto e o nascimento modificaram-se ao longo do tempo. Enquanto antes a mulher paria em seus domicilios com o auxilio de entes queridos ou outras mulheres, posteriormente, com os altos índices de mortalidade materno-infantil, surgiu a necessidade da institucionalização e medicalização do parto. Havendo assim, a transferência do protagonismo da mulher para equipe médica e auxiliares ${ }^{1}$.

Juntamente com a institucionalização, houve um aumento de práticas como intervenções cesáreas, incentivando posteriormente a atuação do profissional de enfermagem através de normatizações legais como a Portaria no 163 , de 22/09/1998, da Secretaria de Assistência à Saúde do Ministério da Saúde, e da Resolução do Conselho Federal de Enfermagem 223/19992.3. Além do Programa de Humanização do Pré-Natal e Nascimento (PHPN) ${ }^{4}$, tendo como principal desígnio assegurar a melhoria do acesso, da cobertura e da qualidade do atendimento pré-natal e da assistência ao parto e puerpério.

Tendo em vista que a enfermagem obstétrica visa boas práticas para mudanças paradigmáticas com um parto humanizado e seguro que devolva o protagonismo da mulher no parto, faz-se necessário a avaliação dessa assistência. Assim sendo, o presente estudo teve como objetivo avaliar a assistência prestada pelas enfermeiras obstetras no processo de parturição através da percepção das mulheres ${ }^{5,6}$.

\section{METODOLOGIA}

Trata-se de um estudo exploratório, descritivo, de abordagem qualitativa. Participaram da pesquisa 28 púerperas que foram assistidas por enfermeiras obstetras durante o trabalho de parto e parto realizados em Maternidade localizada no bairro de Afogados da rede municipal de saúde da cidade do Recife-PE, no período de outubro a novembro de 2016, sendo o número de participantes definidos a partir da saturação do discurso?.

Foram realizadas entrevistas do tipo semiestruturada, gravadas e voltadas ao objetivo principal da pesquisa, buscando identificar qual a visão sobre a assistência da enfermeira obstetra que acompanhou seu parto? Após aceitação e assinatura do termo de consentimento livre e esclarecido (TCLE), a coleta foi realizada no momento do puerpério, onde todas que se encaixaram nos critérios de inclusão foram entrevistadas. As gravações foram transcritas de forma integral e utilizou-se a técnica de análise de conteúdo do tipo temática com o uso da grelha de Bardin para facilitar a interpretação dos dados, onde os núcleos de sentido foram organizados em categorias ${ }^{8}$.

Como critérios de inclusão foram utilizados: ser maior de idade e desejasse participar livremente do estudo e como critérios de exclusão: Intercorrências no trabalho de parto e parto, gestantes de alto risco e puérpera com alguma condição física ou psíquica que impossibilitasse a entrevista. As identidades das participantes foram preservadas, sendo portanto, apresentadas através de nomes de Deusas Gregas, pois estas permeia os mitos com força, coragem e beleza.

$\mathrm{Na}$ pesquisa não houve conflito de interesses e foram levados em consideração os aspectos éticos contidos na Resolução no 466 de 12 de dezembro de 2012 do Conselho Nacional de Saúde (CNS) . A pesquisa foi aprovada pelo comitê de ética do IMIP/PE sob o protocolo $n^{\circ} 1.460 .738$.

\section{RESULTADOS}

As entrevistadas do presente estudo situavam-se na faixa etária de 18 a 39 anos, com média de 23,5 anos de idade. O nível de escolaridade predominante foi ensino médio completo (42,8\%). A maioria declararam união estável (60,7\%) e do $\operatorname{lar}(64,2 \%)$. Residentes da cidade do Recife (82,14\%) e regiões metropolitanas: Jaboatão $(7,1 \%)$ e São Lourenço da Mata (3,5\%) predominaram.

Sobre o pré-natal houve uma média de 6 consultas a maioria em unidades públicas de saúde e 16 relatam orientação acerca do parto e trabalho de parto, porém, notou-se pouco aprofundamento do tema. Quando questionadas qual profissional realizou a assistência ao parto, 18 delas acharam que haviam sido atendidas pelo profissional médico, sendo informadas posteriormente que tinham sido enfermeiras.

No que se refere a visão sobre a assistência da enfermeira obstetra que acompanhou seu parto, sugiram duas idéias centrais, que foram categorizadas de acordo com a grelha de Bardin.

Categoria 1 - Assistência da Enfermeira obstetra que foi dividido em duas subcategorias: 1.1 Sentimentos de Aprovação/satisfação relacionados a assistência prestada pelas enfermeiras obstetras e 1.2 Métodos e Condutas empregados pelas enfermeiras obstetras. Já a categoria 2 referente as experiências de partos anteriores

\section{Assistência do Enfermeiro obstetra:}

1.1. Sentimentos de Aprovação/satisfação relacionados à assistência prestada pelos enfermeiros obstetras:

As entrevistadas declararam muitos medos, preocupações, expectativas e inseguranças que puderam ser amenizadas por uma boa preparação e uma assistência de qualidade.

[...] foi boa! Foi boa! Foi ótima! Ela me deu muita atenção [...] (Afrodite)/ [...]alisando minha barriga, minha cabeça, gostei[...] (Ártemis)/ [...] eu achei assim ele bem ágil, bem cooperativo, 
bem atencioso, bem paciente, me explicou, me auxiliava, tava sempre junto comigo, não só comigo como todo mundo [...] (Hera) / [...]legal! Porque ele ficou o tempo todo comigo! Um mói de coisa, apertando minha mão, mordia ele (risos) [...] (Héstia)

A presença constante do diálogo do profissional com a paciente foi algo percebido, notando-se a importância deste para demonstrações de força durante o parto.

[...] ficou conversando comigo né? Me dando atenção, me explicando...[...] (Atena) / [...] foi ótima. Conversou, me ajudou, me deu força. Me orientou...que eu me acalmasse [...] (Démeter).

Uma analogia positiva foi referida, comparando a enfermeira com algo divino no momento da assistência.

[...] um amor de pessoa. Foi um anjo, uma bença, ela muito paciente, tava toda hora me alisando, pedindo pra eu ter calma, dizendo que ia ser rápido. Foi uma bença, em geral, todos que tavam na sala, foi inexplicável [...] (Gaia) /[...] ela explicou...ela foi bem paciente...todas as horas ela foi paciente...sempre mandando eu ter calma...foi ótimo ela [...] (Nix)

\subsection{Métodos e Condutas empregados pelos enfermeiros obstetras}

A utilização de métodos não farmacológicos também foi presente na verbalização das entrevistadas. A bola, massagens, músicas e danças foram citadas.

[...] me ajudou, me botou na bola... Me ensinou os exercícios também , pra eu ter mais ligeiro[...] (Afrodite)/ [...] as enfermeiras , ó, foi ótimo, as músicas bem baixinha, foi ótimo, ótimo, ótimo[...] (Hémera)/ [...]eles ajudaram, deu assistência. Falavam, eles pegavam, as enfermeiras pegavam assim em mim...(movimento de dança)[...] (Tálassa) /[...] foi ótimo a assistência. Fizeram massagem nas minhas costas[...](Fébi)

Constatamos através dos resultados, que a colocação da parturiente como sujeito esteve presente, fazendo parte inclusive da escolha da posição do parto e de diferentes técnicas utilizadas, cada qual com as suas singularidades e a valorização do protagonismo da mulher.

[...] eles pediram, perguntaram se eu queria deitada ou acocorada, eu preferí acocorada[...] (Mnemósine)/ [...] foi rápido. Eu fiquei sentada num...num negócio pequeno assim baixinho que tinha lá... banqueta? Acho que foi esse [...](Nix)/ [...] ele me ajudou né? Mudando a posição...sempre olhando... gostei[...](Têmis).

Apesar de toda assistência humanizada, ainda encontramos resquícios de uma assistência tecnicista.

[...]Pediram pra fazer força...ai eu fiz a primeira e pediu pra eu respirar fundo[...] (Gaia)

O modelo de assistência obstétrica desempenha um papel de garantia de saúde na gestação, parto e puerpério, trazendo experiências exitosas e humanizadas.(5) Práticas estas muitas vezes atribuídas a classe médica.

[...]a médica fez eu cantar na sala pra eu esquecer a dor (era uma enfermeira) [...](Fébi)/[...]foi ótima, eu pensei até que era uma médica[...](Hemera).

Por outro lado, a enfermeira obstetra recebeu elogios, exaltando e valorizando a atuação e domínio das condutas em questão.

[...] me orientou melhor de que o medico...que me fez um bicho da poxa... ai ele disse que o bebê tava aboiando na barriga...que o cordão podia sair primeiro que o bebê... ela foi e me explicou que não era assim não...que o cordão pode sair antes do bebe em qualquer gestante[...](Réia)

\section{Experiência de partos Anteriores}

A mudança de paradigma dos modelos assistenciais também se faz presente nas falas das mulheres aqui entrevistadas, quando elas se remetem ao passado e comparam ao parto mais recente, reconhecimento este progresso.

[...] E no outro eu não tive tanta assistência como eu tive agora...Agora eu tive gente pra fazer massagem nas minhas costas entendeu?... pra conversar... e no outro não...eu tava sem acompanhante também , mas não teve essa assistência que eu tive agora...[...](Íris)

Há ainda porém, mulheres que não foram preparadas e não reconhecem a violência obstétrica em partos anteriores, apesar de existir.

[...] esse foi mais dor visse? O outro ainda teve ajuda... O médico ele cortou e apertou a minha barriga. Foi mais fácil... dessa foi a força todinha minha[...] (Perséfone)

No estudo em geral, observou-se a ausência de condutas violentas, onde as mulheres atuaram como controladoras do próprio corpo e preservados seus direitos de mulher e paciente, com a escuta de suas queixas e anseios, corroborando com estudo do mesmo tema ${ }^{10}$. Manobras como a episiotomia e a manobra de kristeller relatadas acima, devem ser desencorajadas. ${ }^{11,12}$

Relacionado ao pré-natal, a maioria das entrevistadas apresentam mais de 6 consultas realizadas, número este bem próximo das novas recomendações da OMS. ${ }^{13}$ Entretanto, percebe-se que diversas mulheres não apresentaram um discurso rico em informações, devendo assim serem estimuladas cada vez mais para assumirem uma postura mais independente, ativa e conhecedora dos seus direitos a respeito do parto. ${ }^{6,1,14,15}$

\section{DISCUSSÃO}

A atenção e a companhia da enfermeira, significou ao entendimento delas, uma boa assistência e demonstrou ser 
algo bastante relevante com a sensação de não se sentirem sós.

A enfermagem obstétrica tem um papel fundamental no cuidado e acolhimento as mulheres em trabalho de parto. Incentivando o diálogo, oferecendo apoio, conforto, compreensão, estabelece uma relação de confiança e ameniza o tão temido medo do parto ${ }^{16,17}$

Em relação a comunicação, as palavras devem visar um cuidado individualizado, estratégia esta, de dignificação da enfermagem, e que traz calma e o alivio da dor ${ }^{18} \mathrm{Em}$ contrapartida, a falta desta comunicação destitui da mulher o poder sobre seu próprio corpo, a tornando mais passiva, impedindo assim, sua autonomia e participação ativa no processo de parir ${ }^{19}$

A paciência e calma da equipe de enfermagem também provocaram sentimentos de satisfação, o que contribui para redução de níveis de ansiedade alto antes do parto, como aponta estudo ${ }^{20}$.

A busca por práticas mais humanizadas e acolhedoras, priorizando um cuidado qualificado e com respaldo científico, é uma realidade dentro da prática da enfermagem obstetra ${ }^{21}$.

Estudos corroboram que o uso da analgesia farmacológica pode modificar o parto, aumentando as chances de partos instrumentalizados e de maior duração ${ }^{22}$. A utilização de métodos não farmacológicos e não invasivos contribuem positivamente para a efetivação do parto, alívio das contrações, relaxamento e dilatação ${ }^{17}$. Foram relacionados no estudo, a realização de partos mais rápidos ou a satisfação e aceitação de tê-los presentes nos seus trabalhos de parto.

A enfermeira especialista, tem um papel fundamental na mudança de comportamento das gestantes proporcionandolhes informações sobre recursos e competências (23). Entretanto, existem ainda, dificuldades em relação a desconstrução da falta de autonomia da mulher em relação aos profissionais. Faltando assim, escolhas conscientes e de controle do próprio corpo, indispensáveis nesse processo $18,24,25$

A escolha da posição do parto, perpassa por uma relação livre de qualquer ato de coerção ou autoritarismo e imposições, com escolhas conforme necessidades mais convenientes e confortáveis ${ }^{18,26}$.

A presença de esforços de puxo prolongados e dirigidos, apontados em alguns discursos da pesquisa, representa práticas prejudiciais, pois violam a autonomia e desrespeita o parto como um processo fisiológico, o que prejudica a tomada de decisões mais próximas dos desejos e anseios da mulher ${ }^{27}$.

Estudo refere que assistências envolvendo estes profissionais associam-se a menores taxas de intervenções e em maior satisfação das mulheres. ${ }^{26,28}$ Pesquisa realizada pela Universidade de Oxford concluiu que nenhum país reduziu a mortalidade materna sem investir na capacidade das obstetras e/ou das Enfermeiras Obstetras ${ }^{29}$

O predomínio da enfermagem na assistência obstétrica reforça a sua importante contribuição no que se refere à prática assistencial. Destacam-se em alguns estudos condutas que se apoiam na humanização da assistência, como o direito a acompanhante, liberdade de posição e movimento, utilização de métodos não farmacológicos de alívio da dor, baixa prevalência de episiotomia e de toques vaginais sucessivos, além de se evitar hospitalizações precoces ${ }^{21,28,30}$.

Podemos concluir em algumas falas, a mudança do modelo assistencial entre os partos anteriores e o mais atual de forma positiva. Entretanto, observou-se a falta do acompanhante, embora em poucos discursos, que assegura a mulher, apoio físico e emocional, além de inibir intervenções desnecessárias e violentas ${ }^{31,32}$.

Alguns estudos do mesmo tema, ainda referem desrespeito e violência praticados pela equipe de saúde, nos fazendo refletir que melhorias ainda são necessárias 1,33,34,35.

Como fator limitante do estudo pode-se citar ter sido desenvolvido apenas no município de Recife, necessitando de outros estudos semelhantes em outras regiões no âmbito nacional e mundial.

\section{CONSIDERAÇÕES FINAIS}

Ao concluir o presente estudo, verificamos que a maioria absoluta das puérperas entrevistadas apresentava um bom nível de satisfação em relação à assistência da enfermagem obstétrica. A inserção desta especialidade em espaços de parto proporciona práticas que visam colocar a mulher como sujeito do seu próprio corpo.

O estudo mostra que 6 consultas pré-natais foi a média obtida. Tal consulta, apresenta um papel importantíssimo dentro da condição de empoderamento da mulher quando se trata de obtenção de informações cruciais para esta ter domínio pleno do seu próprio corpo, entendendo todo o processo da gestação ao parto. Há ainda a necessidade de estímulos maiores de troca de saberes, com práticas sempre baseadas nas histórias de vida, suas individualidades e evidências científicas.

É importante cada vez mais a inserção da enfermagem obstétrica nas instituições de ensino e locais de partos, como também em todos os espaços que se encontrem as mulheres, desde as comunidades, unidades básicas de saúde e hospitais de referência. Grupos de gestantes, consultas pré-natais de qualidade, partos humanizados e outros estudos deste tipo devem ser estimulados. 


\section{REFERÊNCIAS}

1. Lima, Welman de Sousa et al. Assistência ao Parto e suas mudanças ao longo do tempo no Brasil. Rev. Multidebates. [internet] 2018 Out. [cited 2018 Nov 15]: 2(2): 41-55, out. 2018. Available from: http://itopedu com.br/revista/index.php/revista/article/view/l17.

2. Ministério da Saúde (Brasil). Portaria número 163, de 22 de setembro de 1998. Diário Oficial da União 24 set 1998 [cited 2017 Fev 15]. Available from: http://sna.saude.gov.br/legisla/legisla/obst/SAS_P163_98obst. doc

3. Conselho Federal de Enfermagem(COFEN). Resolução $n^{\circ} 223$, de 03 de dezembro de 1999. Dispõe sobre a atuação de Enfermeiros na Assistência à mulher no ciclo gravidico Puerperal. Diário Oficial da União 03 jun 1999 [cited 2017 fev 15] Available

\section{from: http://www.cofen.gov.br/resoluo-cofen-2231999_4266.html}

4. Ministério da Saúde (Brasil). Portaria no 569, de 1 de junho de 2000 Dispõe sobre a instituição do Programa de Humanização no Pré-natal e Nascimento no âmbito do Sistema Único de Saúde. Diário Oficial da União 08 jun 2000 [cited 2017 fev 16] Available from: http://bvsms.saude.gov.br/bvs/saudelegis/gm/2000/prt0569_01_06_2000_rep.html

5. Rêgo Midiã Gomes da Silva, Vilela Mirella Bezerra Rodrigues, Oliveira Conceição Maria de, Bonfim Cristine Vieira do. Óbitos perinatais evitáveis por intervenções do Sistema Único de Saúde do Brasil. Rev. Gaúcha Enferm. [Internet]. 2018 [citado 2018 Nov 14] : 39: e2017-0084 Disponivel em: http://www.scielo.br/scielo.php?script=sci_arttext\&pid=S1983-14472018000100414\&lng=pt.

6. Pereira Simone Barbosa, Diaz Claudia Maria Gabert, Backes Marli Terezinha Stein, Ferreira Carla Lizandra de Lima, Backes Dirce Stein. Good practices of labor and birth care from the perspective of health professionals. Rev. Bras. Enferm. [Internet]. 2018 [cited 2018 Nov 25] : 71 Suppl 3 ): 1313-1319. Available from: http://www.scielo.br/scielo.php?script=sci_arttext\&pid=S0034-71672018000901313\&lng=en.

7. Fontanela BJB; Magdaleno Junior R. Saturação teórica em pesquisas qualitativas: contribuições psicanalíticas. Psicol estud. [internet]. 2012 [cited 2017 jan]; 17(1):63-71. Available from: http://www.redalyc.org/articulo.oa?id=287123554008

\section{Bardin L. Análise de conteúdo. Lisboa: Edições 70; 2011.}

9. Conselho Nacional de Saúde (Brasil). Resolução n 466 , de 12 de dezembro de 2012. Diretrizes e normas regulamentadoras de pesquisas envolvendo seres humanos. Diário Oficial da União 13 jun 2013 [cited 2017 Ago 6]; Seção 1. Available from: http://conselho.saude. gov.br/resolucoes/2012/Reso466.pdf.

10. Possati Andrêssa Batista, Prates Lisie Alende, Cremonese Luiza Scarton Juliane, Alves Camila Neumaier, Ressel Lúcia Beatriz. Humanização do parto: significados e percepções de enfermeiras. Esc Anna Nery [Internet]. 2017 [cited 2018 Nov 25] : 21( 4 ): e20160366. Available from: http://www.scielo.br/scielo.php?script=sci_arttextEpi$d=S 1414-81452017000400203$ \&lng=en

11. Cunha et al. Posição da parturiente durante o parto vaginal: divergência entre a prática médica e de enfermagem. Rev. Bras. Pesq. Saúde. 2[internet]. 2017 Out. [cited 2018 Nov 20]; 19(4): 58-64. Available from http://periodicos.ufes.br/RBPS/article/view/19804/13226

12. Leal Maria do Carmo, Pereira Ana Paula Esteves, Domingues Rosa Maria Soares Madeira, Filha Mariza Miranda Theme, Dias Marcos Augusto Bastos, Nakamura-Pereira Marcos et al. Obstetric interventions during labor and childbirth in Brazilian low-risk women. Cad. Saúde Pública [Internet]. 2014 [cited 2018 Nov 25] ; 30( Suppl 1 ): S17-S32. Available from: http://www.scielo.br/scielo.php?script=sci_arttext\&pi$d=S 0102-311 \times 2014001300005 \&$ ing $=e n$.
13. Organização Mundial de Saúde(OMS). Recomendações da OMS sobre cuidados pré-natais para uma experiência positiva na gravidez. [internet] 2016. [cited 2017 Fev 20]. Available from: https://www.who.int/ reproductivehealth/publications/maternal_perinatal_health/anc-positive-pregnancy-experience/en/

14. Silva DF, Peres LC, Araújo NCS. Conhecimento das gestantes sobre as posições do parto. Refaci. [internet] 2018 Jan. [cited 2018 Nov 20] 1(1): 1-9. Available from: http://revista.faciplac.edu.br/index.php/REFACI/article/view/504/192

15. Kottwitz Fernanda, Gouveia Helga Geremias, Gonçalves Annelise de Carvalho. Via de parto preferida por puérperas e suas motivações. Esc. Anna Nery [Internet]. 2018 [citado 2018 Nov 25] : 22( 1 ): e20170013. Disponivel em: http://www.scielo.br/scielo.php?script=sci_arttext\&pi$\mathrm{d}=$ S1414-814520180001002018lng=pt. Epub 17-Nov-2017.

16. Carvalho Silas Santos, Oliveira Bruno Rodrigues de, Nascimento Camila Silva Oliveira do, Gois Carla Tatiane de Souza, Pinto Iasmim Oliveira. Perception of a nursing team in the implantation of a reception with risk classification sector for pregnant women. Rev. Bras. Saude Mater Infant. [Internet]. 2018 June [cited 2018 Nov 25] ; 18( 2 ): 301-307. Available from: http://www.scielo.br/scielo.php?script=sci_arttext\&pi$d=S 1519-38292018000200301 \& \operatorname{lng}=e n$

17. Silva IA, Silva PSF, Andrade EOF, Morais FF, Silva RSS, Oliveira LS Percepção das puérperas acerca da assistência de enfermagem no parto humanizado. Rev Uningá. [internet]. 2017 jul.[cited 2018 nov 12] ;53(2): 37-43. Available from: https://www.mastereditora.com.br/periodico/20170806_102009.pdf

18. Silva Andréa Lorena Santos, Nascimento Enilda Rosendo do, Coelho Edméia de Almeida Cardoso. Práticas de enfermeiras para promoção da dignificação, participação e autonomia de mulheres no parto normal. Esc. Anna Nery [Internet]. 2015 Sep [cited 2018 Nov 25] : 19( 3 ): 424431. Available from: http://www.scielo.br/scielo.php?script=sci_arttextEpid=S1414-81452015000300424\&lng=en.

19. Scarton Juliane, Prates Lisie Alende, Wilhelm Lais Antunes, Silva Silvana Cruz da, Possati Andressa Batista, Ilha Caroline Bolzan et al. "No final compensa ver o rostinho dele": vivências de mulheres-primiparas no parto normal. Rev. Gaúcha Enferm. [Internet]. 2015 [cited 2018 Nov 25] ; 36( spe ): 143-151. Available from: http://www.scielo.br/scielo. php?script=sci_arttext\&pid=S1983-14472015000500143\&lng=en.

20. Molina-Fernández Inmaculada de, Rubio-Rico Lourdes, Roca-Biosca Alba, Jimenez-Herrera Maria, Flor-Lopez Miriam de la, Sirgo Agustina. Ansiedad y miedos de las gestantes ante el parto: La importancia de su detección. Revista Portuguesa de Enfermagem de Saúde Mental [Internet]. 2015 Jun [citado 2018 Nov 26] : ( 13 ): 17-24. Disponivel em: http://www.scielo.mec.pt/scielo.php?script=sci_arttext\&pi$d=S 1647-21602015000200003 \&$ lng $=p t$.

21. Medeiros Renata Marien Knupp, Teixeira Renata Cristina, Nicolini Ana Beatriz, Alvares Aline Spanevello, Corrêa Áurea Christina de Paula, Martins Débora Prado. Cuidados humanizados: a inserção de enfermeiras obstétricas em um hospital de ensino. Rev. Bras. Enferm. [Internet]. 2016 Dec [cited 2018 Nov 25] ; 69( 6 ): 1091-1098. Available from: http://www.scielo.br/scielo.php?script=sci_arttext\&pi$d=$ S0034-716720160006010918lng=en

22. Felisbino-Mendes Mariana Santos, Santos Luiza Oliveira, Amorim Torcata, Costa Isabela Nascimento, Martins Eunice Francisca. O uso de analgesia farmacológica influencia no desfecho de parto?. Acta paul. enferm. [Internet]. 2017 Oct [cited 2018 Nov 25] ; 30( 5 ): 458-465. Available from: http://www.scielo.br/scielo.php?script=sci_arttext\&pi$d=S 0103-21002017000500458 \&$ ing=en.

23. Martins Maria de Fátima da Silva Vieira, Remoaldo Paula Cristina Almeida Cadima. Representações da enfermeira obstetra na perspetiva 
da mulher grávida. Rev. bras. enferm. [Internet]. 2014 June [cited 2018 Nov 25] ; 67( 3 ): 360-365. Available from: http://www.scielo.br/scielo. php?script=sci_arttextEpid=S0034-71672014000300360\&lng=en.

24. Reis Thamiza Laureany da Rosa dos, Padoin Stela Maris de Mello, Toebe Thayla Rafaella Pasa, Paula Cristiane Cardoso de, Quadros Jacqueline Silveira de. Autonomia feminina no processo de parto e nascimento: revisão integrativa da literatura. Rev. Gaúcha Enferm. [Internet]. 2017 [cited 2018 Nov 25] ; 38(1): e64677. Available from: http://www.scielo.br/ scielo.php?script=sci_arttext\&pid=S1983=14472017000100503-\&lngen.

25. Kottwitz Fernanda, Gouveia Helga Geremias, Gonçalves Annelise de Carvalho. Via de parto preferida por puérperas e suas motivações. Esc. Anna Nery [Internet]. 2018 [citado 2018 Nov 25] ; 22( 1): e20170013. Disponivel em: http://www.scielo.br/scielo.php?script=sci_arttextEpi$d=$ S1414-81452018000100201Elng=pt.

26. Nascimento Raquel Ramos Pinto do, Arantes Sandra Lucia, Souza Eunice Delgado Cameron de, Contrera Luciana, Sales Ana Paula Assis. Escolha do tipo de parto: fatores relatados por puérperas. Rev. Gaúcha Enferm. [Internet]. 2015 [cited 2018 Nov 25] ; 36 (spe ): 119-126. Available from: http://www.scielo.br/scielo.php?script=sci_arttext\&pi$d=$ S1983-14472015000500119\&ing=en.

27. Reis Carolyn Cristina, Ferreira de Souza Karla Romana, Santos Alves Danielle, Tenório Inez Maria, Brandão Neto Waldemar. Percepção das mulheres sobre a experiência do primeiro parto: Implicações para o cuidado de Enfermagem .[internet]. 2016 out. [ cited 2018 nov 15] : 23( 2 ): 45-56. Available from: https://scielo.conicyt.cl/scielo.php?script=sci_arttext\&pid=S0717-95532017000200045\&lng=e.

28. Sousa Ana Maria Magalhães, Souza Kleyde Ventura de, Rezende Edna Maria, Martins Eunice Francisca, Campos Deise, Lansky Sônia. Práticas na assistência ao parto em maternidades com inserção de enfermeiras obstétricas, em Belo Horizonte, Minas Gerais. Esc. Anna Nery [Internet]. 2016 June [cited 2018 Nov 25] ; 20 ( 2 ): 324-331. Available from: http://www.scielo.br/scielo.php?script=sci_arttextEpi$\mathrm{d}=$ S1414-814520160002003248lng=en.

29. Organización de los Estados Americanos (OEA). Acceso a servicios de salud materna desde una perspectiva de derechos humanos. [internet] 2010 jun.[citado 2017 fev 20]. Available from: http://cidh.org/women/saludmaternalOsp/SaludMaterna2010.pdf
30. Possati Andrêssa Batista, Prates Lisie Alende, Cremonese Luiza, Scarton Juliane, Alves Camila Neumaier, Ressel Lúcia Beatriz. Humanização do parto: significados e percepções de enfermeiras. Esc. Anna Nery [Internet]. 2017 [cited 2018 Nov 25] : 21( 4 ): e20160366. Available from: http://www.scielo.br/scielo.php?script=sci_arttext\&pi$d=S 1414-81452017000400203 \& \operatorname{lng}=e n$.

31. Rodrigues Diego Pereira, Alves Valdecyr Herdy, Penna Lucia Helena Garcia, Pereira Audrey Vidal, Branco Maria Bertilla Lutterbach Riker, Souza Rosangela de Mattos Pereira de. O descumprimento da lei do acompanhante como agravo à saúde obstétrica. Texto contexto - enferm. [Internet]. 2017 [cited 2018 Nov 25] :26(3):e5570015. Available from: http://www.scielo.br/scielo.php?script=sci_arttextepi$d=$ S0104-07072017000300319\&lng=en.

32. Brüggemann Odaléa Maria, Ebsen Erika Simas, Ebele Romana Raquel, Batista Bruna Daniela. Possibilidades de inserção do acompanhante no parto nas instituições públicas. Ciênc. saúde coletiva [Internet]. 2016 Aug [cited 2018 Nov 25] : 21( 8 ): 2555-2564. Available from: http://www.scielo.br/scielo.php?script=sci_arttextEpi$d=S 1413-81232016000802555$ \&lng=en.

33. Oliveira Virgínia Junqueira, Penna Cláudia Maria de Mattos. O discurso da violência obstétrica na voz das mulheres e dos profissionais de saúde. Texto contexto - enferm. [Internet]. 2017 [cited 2018 Nov 25] 26( 2 ): e06500015. Available from: http://www.scielo.br/scielo.php?script=sci_arttextEpid=S0104-07072017000200331Elng=en.

34. de Cássia Versiani, C, Barbieri, M, Gabrielloni, MC, Fustinoni, SM. Significado de parto humanizado para gestantes. Revista de Pesquisa Cuidado é Fundamental Online [Internet]. 2015 jan [cited $2017 \mathrm{Fev}$ 14]:7(1):1927-1935. Available from: http://www.redalyc.org/articulo. oa?id $=505750945017$

35. Silva RM, Farias MCAD, Sousa AKA, Abreu RMSX, Silva RA. Atuação da enfermagem em alojamento conjunto: percepção de puérperas. REBES. [internet] 2015 Jul. [cited 2018 Nov 14] ;5(3): 35-44. Available from: https://www.gvaa.com.br/revista/index.php/REBES/article/ view/3664/3311 